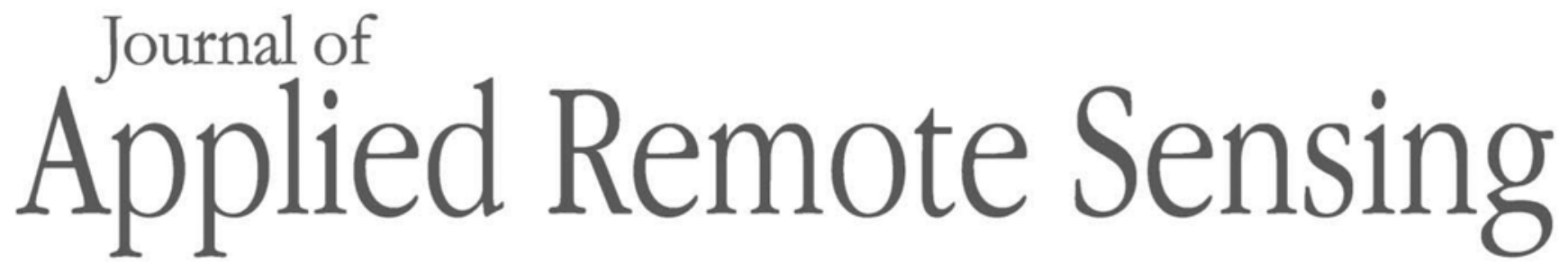

RemoteSensing.SPIEDigitalLibrary.org

\title{
Detecting and monitoring water stress states in maize crops using spectral ratios obtained in the photosynthetic domain
}

Gladimir V. G. Baranoski

Spencer R. Van Leeuwen 


\title{
Detecting and monitoring water stress states in maize crops using spectral ratios obtained in the photosynthetic domain
}

\author{
Gladimir V. G. Baranoski*, and Spencer R. Van Leeuwen \\ University of Waterloo, David R. Cheriton School of Computer Science, \\ Natural Phenomena Simulation Group, Waterloo, Ontario, Canada
}

\begin{abstract}
The reliable detection and monitoring of changes in the water status of crops composed of plants like maize, a highly adaptable $\mathrm{C} 4$ species in large demand for both food and biofuel production, are longstanding remote sensing goals. Existing procedures employed to achieve these goals rely predominantly on the spectral signatures of plant leaves in the infrared domain where the light absorption within the foliar tissues is dominated by water. It has been suggested that such procedures could be implemented using subsurface reflectance to transmittance ratios obtained in the visible (photosynthetic) domain with the assistance of polarization devices. However, the experiments leading to this proposition were performed on detached maize leaves, which were not influenced by the whole (living) plant's adaptation mechanisms to water stress. In this work, we employ predictive simulations of light-leaf interactions in the photosynthetic domain to demonstrate that the living specimens' physiological responses to dehydration stress should be taken into account in this context. Our findings also indicate that a reflectance to transmittance ratio obtained in the photosynthetic domain at a lower angle of incidence without the use of polarization devices may represent a cost-effective alternative for the assessment of water stress states in maize crops. () The Authors. Published by SPIE under a Creative Commons Attribution 3.0 Unported License. Distribution or reproduction of this work in whole or in part requires full attribution of the original publication, including its DOI. [DOI: 10.1117/1. JRS.11.036025]
\end{abstract}

Keywords: foliar optical properties; water stress; chloroplast relocation; simulation.

Paper 170447P received May 25, 2017; accepted for publication Aug. 24, 2017; published online Sep. 16, 2017.

\section{Introduction}

In recent years, we have been observing an accentuation of adverse climatic conditions leading to extensive drought periods in certain regions of the planet. At the same time, the demand for increasing crop yields to support both food and biofuel production continues to grow steadily. The combination of these factors has become a catalyst for research efforts toward the effective detection and monitoring of changes in crops' water status. These procedures are essential not only from an economical but also from an ecological point of view. An underestimation of crops' water requirements may lead to reduced yields, while an overestimation may result in detrimental effects to the environment and human health, such as the contamination (e.g., due to the excessive use of fertilizers) and even depletion of limited freshwater supplies. ${ }^{1}$

Remote sensing initiatives combined with the acquisition and analyses of ground-based spectral data for plants have a central role in this scenario. They can provide valuable support for the development of reliable procedures and sensors for detecting dehydration stress. These, in turn, can be instrumental in limiting irrigation water overuse and preventing long-term yield reductions. Since monocotyledonous $C_{4}$ species characterized by unifacial leaves, such as maize (Zea mays $L$; corn), not only are large-scale providers of raw materials for food and biofuel production but also are endowed with advanced mechanisms for adaptation to adverse environmental conditions, they have been an object of a wide range of experimental investigations in this area. ${ }^{2-7}$

*Address all correspondence to: Gladimir V. G. Baranoski, E-mail: gvgbaran@cs.uwaterloo.ca 
Despite these efforts, however, the current understanding about these species' capacity to grow in hot climates with sporadic rainfall is still relatively limited.

The assessment of a plant's water status is associated with the measurement of its leaves' relative water content (RWC). According to Loreto et al., ${ }^{8}$ when the RWC falls below 70\%, irreversible damage of the foliar photosynthetic apparatus can result. More specifically, the RWC reduction is accompanied by an accentuated drop in pigment content. ${ }^{9,10}$ This reduces light absorption efficiency, which may lead to the plant's death. ${ }^{8,11}$ Moderate water stress (RWC $>70 \%$ ), which is observed more often in nature, ${ }^{8}$ may also prompt structural variations, such as tissue thickness reductions, ${ }^{12}$ that can affect foliar optical properties and, consequently, the leaves' spectral signatures.

Although the effective monitoring of maize crops' water status requires the detection of changes in foliar spectral responses under in vivo conditions, the spectral data used in studies involving the reduction of foliar water content, notably in the more conspicuous moderate range, are normally obtained under in vitro conditions. In these situations, in which the specimens (leaves) are usually detached from a living plant and then air dried, an increase in the visible reflectance is observed. ${ }^{2,13}$ One might expect that in vivo and in vitro water reduction procedures would result in similar spectral changes since, in both cases, the loss of water affects the internal structure of the foliar tissues. However, experiments performed by Maracci et al. ${ }^{3}$ under in vivo conditions (by withholding water from the soil), which were also examined by Verdebout et al., ${ }^{4}$ suggested that this may not be the case for certain species. More specifically, in these experiments, maize leaves whose water content was moderately reduced using an in vivo procedure showed a decrease in the visible reflectance even though their pigment content remained relatively constant. ${ }^{3}$

According to Loretto et al., ${ }^{8}$ during slowly developing water stress (e.g., when plants sense the reduction of soil water content), water deficit signals are released in the live plant roots and delivered to the leaves. The main signaling route used in this process is mediated by abscisic acid (ABA). ${ }^{14}$ It is worth noting that, as ABA is detected, it induces cytosolic $\mathrm{Ca}^{2+}$ elevations. ${ }^{8}$ Also, $\mathrm{Ca}^{2+}$ channels are essential for the initiation of a large number of signal transduction processes in higher plant cells. ${ }^{15}$ For example, experiments by Sato et al. ${ }^{16}$ indicate that the influx of external $\mathrm{Ca}^{2+}$ through the plasma membrane is required for the early signaling step of chloroplast mechanorelocation movements. Accordingly, it has been proposed that a similar sequence of events may occur in maize leaves under moderate in vivo water stress and neutral illumination conditions. ${ }^{7}$ More specifically, an elevation of cytosolic $\mathrm{Ca}^{2+}$, induced by water deficit signals, might provide a signaling pathway to trigger the migration of chloroplasts away from the walls of the cells, which, in turn, might result in a more homogeneous distribution of these chlorophyllcontaining organelles. Such a distribution pattern increases the probability of light detour effects and, consequently, light absorption in the visible (photosynthetic) domain. ${ }^{17,18}$ This may explain the reflectance decrease observed during moderate in vivo water reduction procedures, and it may also represent further evidence about the adaptive mechanisms of these plants to adverse environmental conditions., ${ }^{5,6}$

Estimations of plants' water content usually involve spectral measurements in the infrared domain. ${ }^{19,20}$ However, a number of studies have addressed the possibility of using foliar spectral signatures in the photosynthetic domain to assist in the assessment of a plant's water status. ${ }^{13,21,22}$ Vanderbilt et al. $^{23}$ performed experiments in which optical polarization techniques were employed to remove the surface (specular) component of maize leaves' reflectance to demonstrate that its subsurface (diffuse) component has a direct correlation with the leaves' RWC. This correlation was based on the premise that RWC-linked changes in the visible reflectance are associated with structural alterations in the internal arrangement of the foliar tissues and are not a consequence of molecular configuration changes in cellular pigments, provided that the leaves' RWC is above $65 \%$. Taking this correlation into account, Vanderbilt et al. ${ }^{23}$ indicated that the subsurface reflectance to transmittance ratio $\left(\rho_{\mathrm{d}} / \tau\right)$ can potentially be used to estimate the RWC of wilted specimens, notably for RWC values between $85 \%$ and $65 \%$. It is important to note, however, that these experiments were performed on leaves detached from the living plant, i.e., under in vitro conditions.

Computer simulations, or in silico experiments, are routinely being employed to accelerate the different cycles of research involving optical processes that cannot be fully studied through 
traditional laboratory procedures due to technical limitations. Among these limitations, one can highlight the difficulties of performing in situ measurements requiring the control of a large number of biophysical variables. In the case of plants, it has been demonstrated that computer simulations paired with measured data can be effectively employed in the investigation of open questions involving their physiological responses to changes in water soil levels, ${ }^{7}$ as well as the effects of limited water availability on their growing process. ${ }^{24}$

In this paper, which is an updated and extended version of a conference presentation, ${ }^{25}$ we employ computer simulations to assess whether variations on the visible spectral signatures of maize leaves can be effectively employed in the detection and monitoring of RWC changes under both in vitro and in vivo conditions. Our simulations are performed using the predictive model of light-leaf interactions known as ABM-U [algorithmic BDF (bidirectional scattering distribution function) model for unifacial leaves $].^{26,27}$ Besides enabling us to overcome the technical limitations mentioned above, the use of such a model allows us to obtain the subsurface component of foliar reflectance in a straightforward manner. Our findings confirm that, when designing procedures for the estimation of changes in maize leaves' water status, one needs to account for the living specimens' physiological responses to dehydration stress. They also indicate that a visible reflectance to transmittance ratio can be potentially used as an indicator of moderate water stress in maize plants under in vivo conditions. This, in turn, can contribute to the prevention of irreversible damages to their photosynthetic apparatus and consequent reductions in crop yield.

\section{Materials and Methods}

\subsection{Simulation Framework Overview}

The ray-optics based model, ABM-U, ${ }^{26,27}$ used in this investigation employs an algorithmic Monte Carlo formulation that provides a rigorous and yet flexible approach for the simulation of light transport within foliar tissues. Its detailed parameter space enables specific biophysical characteristics of different specimens to be appropriately taken into account during the simulations. It is important to note that this model has been extensively evaluated through quantitative and qualitative comparisons of modeled results with actual measured data. ${ }^{7,26,28}$

Within the ABM-U formulation, a ray interacting with a given leaf specimen can be associated with any selected wavelength within the spectral regions of interest. Hence, ABM-U can provide reflectance and transmittance quantities with different spectral resolutions. For consistency, however, we considered a spectral resolution of $5 \mathrm{~nm}$ in all modeled curves depicted in this work. In terms of illumination and collection geometries, ABM-U can provide bidirectional reflectance and transmittance values by recording the direction of the outgoing rays using a virtual gonioreflectometer. ${ }^{29,30}$ In addition, one can obtain directional-hemispherical reflectance and transmittance values by integrating the outgoing rays with respect to the collection hemisphere using a virtual spectrophotometer. ${ }^{30,31}$ Unless, otherwise stated, the spectral curves presented in this investigation correspond to directional-hemispherical readings obtained considering an angle of incidence equal to $8 \mathrm{deg}$ (for consistency with experiments aimed at remote sensing observations of plants ${ }^{32}$ ) and $10^{6}$ sample rays per wavelength.

To enable the full reproduction of our investigation results, we made ABM-U available online $^{33}$ via a model distribution system ${ }^{34}$ along with the supporting biophysical data ${ }^{35}$ (e.g., refractive indices and extinction coefficients) used in our in silico experiments. This system enables researchers to specify experimental conditions (e.g., angle of incidence and spectral range) and specimen characterization parameters (e.g., pigments and water content) using a web interface (Fig. 1) and to receive customized simulation results.

\subsection{Specimens' Characterization Data}

Three groups of maize specimens were considered in this investigation, namely fresh (turgid, baseline), in vitro wilted, and in vivo wilted. Moreover, within each group, we also considered exemplars, henceforth referred to as A1, A2, B1, and B2, with distinct biophysical 


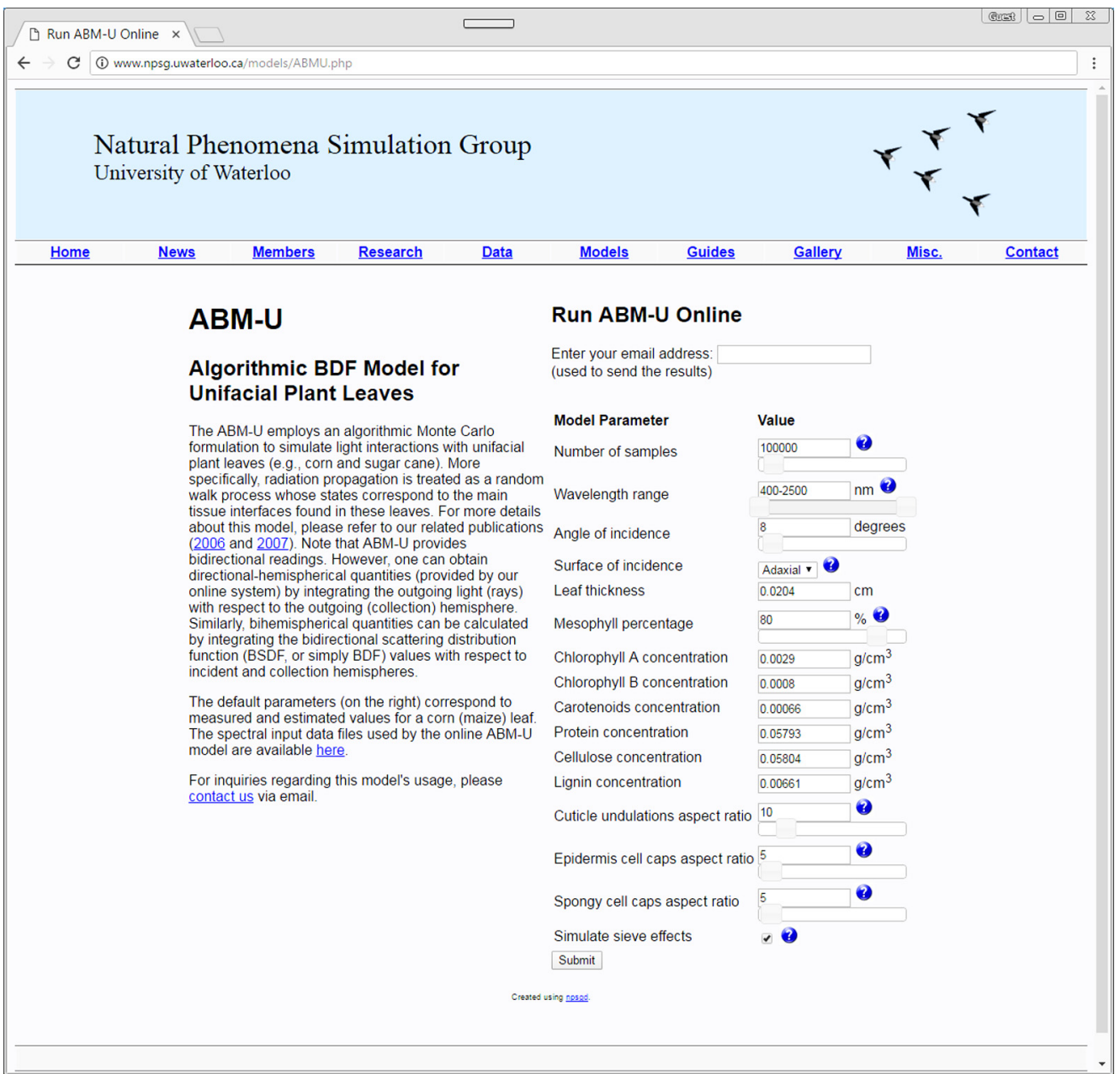

Fig. 1 The web interface for the ABM-U mode $\left.\right|^{33}$ available through the Natural Phenomena Simulation Group Distributed (NPSGD) system. ${ }^{34}$ Through this interface, researchers can configure biophysical parameters and execute light transport simulations involving $\mathrm{C}_{4}$ plants, such as maize, characterized by unifacial plant leaves.

characteristics. We used measured datasets, which are available in the LOPEX database,$^{32}$ in the characterization of the four fresh specimens and modified versions of these datasets in the characterization of the corresponding wilted specimens. These datasets and their modifications are reported in the remainder of this section.

The LOPEX project ${ }^{32}$ involved experiments performed on 120 leaf samples representative of more than 50 species. Specimens A1 and A2 considered in this investigation correspond to LOPEX sample 12, while specimens B1 and B2 correspond to LOPEX sample 16. Each LOPEX sample comprised $\sim 800 \mathrm{~g}$ of collected leaves. The LOPEX experiments included directional-hemispherical reflectance and transmittance measurements, as well as auxiliary measurements of pigment concentrations, thickness, and water content for each specimen. Again, it is important to note that the modeled (using the ABM-U) reflectance and transmittance curves obtained for the baseline specimens considered in this investigation closely approximate their measured LOPEX counterparts as depicted in previous works. ${ }^{7,26}$

The LOPEX biochemical data, namely the contents of the main leaf absorbers in the visible range, used to characterize the baseline (fresh) specimens are presented in Table 1. The remaining LOPEX data employed in the characterization of these specimens, namely thickness, fresh weight, and dry weight, are presented in Table 2 . It is worth noting that the data provided in Tables 1 and 2 were obtained from leaf specimens collected during the period of maximum phenological activity of the sampled plants. The values for thickness, fresh weight, and dry weight parameters correspond to measurements performed for each specimen considering 
Table 1 Biochemical data for specimens associated with LOPEX samples 12 (A1 and A2) and 16 (B1 and B2). The concentration of chlorophyll a $\left(C_{\mathrm{a}}\right)$, chlorophyll b $\left(C_{\mathrm{b}}\right)$, and carotenoids $\left(C_{\mathrm{c}}\right)$ is given as content per fresh weight. Protein $\left(C_{\mathrm{p}}\right)$, cellulose $\left(C_{\mathrm{cl}}\right)$, and lignin $\left(C_{\mathrm{l}}\right)$ contents are given in terms of percentage of dry weight.

\begin{tabular}{lcccccc}
\hline \hline Specimens & $C_{\mathrm{a}}\left(\mathrm{mg} \mathrm{g}^{-1}\right)$ & $C_{\mathrm{b}}\left(\mathrm{mg} \mathrm{g}^{-1}\right)$ & $C_{\mathrm{c}}\left(\mathrm{mg} \mathrm{g}^{-1}\right)$ & $C_{\mathrm{p}}(\%)$ & $C_{\mathrm{cl}}(\%)$ & $C_{\mathrm{l}}(\%)$ \\
\hline A1 and A2 & 2.90 & 0.80 & 0.66 & 26.55 & 26.60 & 3.03 \\
B1 and B2 & 3.16 & 1.11 & 0.84 & 24.09 & 25.89 & 2.75 \\
\hline \hline
\end{tabular}

Table 2 Thickness and weight values for specimens associated with LOPEX samples 12 (A1 and A2) and 16 (B1 and B2). These values correspond to the biophysical characteristics of the actual specimens used to obtain the LOPEX spectral measurements 141, 147, 537, and 539, respectively.

\begin{tabular}{lccc}
\hline \hline Specimen & Thickness $(\mathrm{cm})$ & Fresh weight $(\mathrm{g})$ & Dry weight $(\mathrm{g})$ \\
\hline A1 & 0.0204 & 0.0668 & 0.0146 \\
A2 & 0.0186 & 0.0688 & 0.0170 \\
B1 & 0.0224 & 0.0796 & 0.0203 \\
B2 & 0.0156 & 0.0595 & 0.0162 \\
\hline \hline
\end{tabular}

a foliar area of $4.1 \mathrm{~cm}^{2}$. These parameters are used to calculate the concentration of the absorbers in terms of since their contents are given either in terms of $\mathrm{mg}$ per fresh weight (in the case of chlorophylls and carotenoids) or as percentage of dry weight (in the case of cellulose, lignin, and protein) in the LOPEX database. The volume considered in these concentration calculations corresponds to the sampled foliar area multiplied by the thickness of the mesophyll tissue, which we estimated to be $\sim 80 \%$ of the leaves' total thickness. ${ }^{36,37}$ The upper bound values selected for the cuticle undulations, epidermis cell caps, and mesophyll (spongy) cell caps used to perturb the light rays interacting with the foliar tissues ${ }^{26}$ were 10,5 , and 5, respectively. These values were derived from data available in the literature and also borne out by observations of cross sections of maize leaves. ${ }^{7}$

The modeled radiometric quantities for the wilted specimens were obtained considering a $25 \%$ water reduction accompanied by minor changes in the pigment contents, as reported in the experiments by Maracci et al., ${ }^{3}$ involving maize leaves under moderate water stress. Measurements performed by Wolley ${ }^{38}$ on maize leaves indicate that such a water content reduction is followed by approximately a $20 \%$ reduction in thickness and a $2 \%$ reduction in area. We reduced the thickness and area of our baseline specimens accordingly, and we performed a $25 \%$ reduction on their fresh weights. A summary of ABM-U parameter values employed in this investigation is provided in Tables 3 and 4 . Note that, as expected, ${ }^{3}$ the performed morphological changes resulted in the pigment concentrations remaining relatively unchanged. Finally, we applied a $20 \%$ increase in the aspect ratio of the mesophyll (spongy) cell caps to account for their resulting flattening. ${ }^{12,26}$

We remark that, as the angular distribution of light transmitted through plant leaves increases, the probability of light absorption increases as well due to the detour effect. ${ }^{18}$ However, it is also necessary to consider that a nonhomogeneous distribution of pigments (under normal conditions, the mesophyll chloroplasts usually remain arrayed along the cell walls ${ }^{39,40}$ ) can reduce the probability of light absorption. ${ }^{41}$ In this situation, light can be propagated without encountering these organelles, a phenomenon known as the sieve effect. ${ }^{40,42}$ Hence, simulations of light transport within foliar tissues need to account for the inverse dependence of detour and sieve effects on the distribution of these absorbers ${ }^{18}$ and on the angular deviations of light travelling in the mesophyll tissue. $^{27}$

To account for the inverse angular relationship of the sieve and detour effects, ABM-U adjusts the ray propagation angle using a bound derived from applied optics experiments. ${ }^{27}$ 
Table 3 Summary of ABM-U parameters employed in the characterization of the maize specimens A1 and A2 (LOPEX sample 12) considered in this investigation. The wilted states correspond to a $25 \%$ reduction in water content.

\begin{tabular}{lcccc}
\hline \hline Parameter & A1 (fresh) & A1 (wilted) & A2 (fresh) & A2 (wilted) \\
\hline Thickness $(\mathrm{cm})$ & 0.02040 & 0.01632 & 0.01860 & 0.01488 \\
Mesophyll percentage (\%) & 80 & 80 & 80 & 80 \\
Chlorophyll A concentration $\left(\mathrm{g} / \mathrm{cm}^{3}\right)$ & 0.00290 & 0.00277 & 0.00328 & 0.00313 \\
Chlorophyll B concentration $\left(\mathrm{g} / \mathrm{cm}^{3}\right)$ & 0.00080 & 0.00076 & 0.00090 & 0.00086 \\
Carotenoids concentration $\left(\mathrm{g} / \mathrm{cm}^{3}\right)$ & 0.00066 & 0.00063 & 0.00075 & 0.00071 \\
Protein concentration $\left(\mathrm{g} / \mathrm{cm}^{3}\right)$ & 0.05793 & 0.07389 & 0.07407 & 0.09425 \\
Cellulose concentration $\left(\mathrm{g} / \mathrm{cm}^{3}\right)$ & 0.05804 & 0.07403 & 0.07421 & 0.09443 \\
Lignin concentration $\left(\mathrm{g} / \mathrm{cm}^{3}\right)$ & 0.00661 & 0.00844 & 0.00845 & 0.01076 \\
Cuticle undulations aspect ratio & 10 & 10 & 10 & 10 \\
Epidermal cell caps aspect ratio & 5 & 5 & 5 & 5 \\
Spongy cell caps aspect ratio & 5 & 6 & 5 & 6 \\
\hline \hline
\end{tabular}

Table 4 Summary of ABM-U parameters employed in the characterization of the maize specimens B1 and B2 (LOPEX sample 16) considered in this investigation. The wilted states correspond to a $25 \%$ reduction in water content.

\begin{tabular}{lcccc}
\hline \hline Parameter & B1 (fresh) & B1 (wilted) & B2 (fresh) & B2 (wilted) \\
\hline Thickness $(\mathrm{cm})$ & 0.02240 & 0.01792 & 0.01560 & 0.01248 \\
Mesophyll percentage (\%) & 80 & 80 & 80 & 80 \\
Chlorophyll A concentration $\left(\mathrm{g} / \mathrm{cm}^{3}\right)$ & 0.00342 & 0.00323 & 0.00367 & 0.00352 \\
Chlorophyll B concentration $\left(\mathrm{g} / \mathrm{cm}^{3}\right)$ & 0.00120 & 0.00115 & 0.00129 & 0.00124 \\
Carotenoids concentration $\left(\mathrm{g} / \mathrm{cm}^{3}\right)$ & 0.00091 & 0.00087 & 0.00098 & 0.00093 \\
Protein concentration $\left(\mathrm{g} / \mathrm{cm}^{3}\right)$ & 0.06656 & 0.08490 & 0.07627 & 0.09728 \\
Cellulose concentration $\left(\mathrm{g} / \mathrm{cm}^{3}\right)$ & 0.07152 & 0.09124 & 0.08197 & 0.10454 \\
Lignin concentration $\left(\mathrm{g} / \mathrm{cm}^{3}\right)$ & 0.00760 & 0.00969 & 0.00870 & 0.01110 \\
Cuticle undulations aspect ratio & 10 & 10 & 10 & 10 \\
Epidermal cell caps aspect ratio & 5 & 5 & 5 & 5 \\
Spongy cell caps aspect ratio & 5 & 6 & 5 & 6 \\
\hline \hline
\end{tabular}

Accordingly, we kept this bound in place (see sieve effects checkbox in Fig. 1) during the simulations involving fresh specimens. For the simulations involving the corresponding in vitro and in vivo wilted specimens, we employ the same modified biophysical datasets (Tables 3 and 4) and model running parameters for both, with the exception of this bound, which we removed for the latter. The removal of this bound resulted in sieve effects not being considered for these specimens. This choice was based on the putative intensification of the detour effects caused by a more homogeneous intracellular distribution of mesophyll chloroplasts in response to water stress. ${ }^{7}$ 
Finally, we applied the law of Gladstone and Dale ${ }^{7}$ to obtain the spectral refractive index of mesophyll cell walls after the water content reduction. Although this adjusted spectral refractive index was considered in the simulations presented in this paper for completeness, we note that its impact in the modeled results was minor.

\section{Results and Discussion}

Initially, we computed the subsurface reflectance and transmittance for the three groups of specimens. As can be observed in Fig. 2, while the subsurface reflectance of the in vitro wilted specimens increased in comparison with the subsurface reflectance of the fresh specimens, a behavior consistent with the in vitro experiments performed by Vanderbilt et al., ${ }^{23}$ the subsurface reflectance of the in vivo wilted specimens decreased, a behavior consistent with the in vivo experiments performed by Maracci et al. ${ }^{3}$ On the other hand, as can be observed in Fig. 3, the transmittance of both groups of wilted specimens, in vitro and in vivo, increased in comparison with the transmittances computed for the fresh specimens. These observations indicate that subsurface reflectance alone cannot be used in RWC estimations since some species, like maize, may adapt to moderate water stress conditions. Such an adaptation, in turn, may result in a decrease in the amount of reflected light instead of an increase. These different reflectance variation patterns may render RWC estimations unreliable. Hence, to detect and monitor moderate water stress conditions before damage to the plants' photosynthetic apparatus becomes irreversible, it may be necessary to acquire supporting ground-based information, including both reflectance and transmittance data.

At first glance, it might seem that the use of transmittance readings could be sufficient for obtaining reliable RWC estimations since this spectral quantity increased for both groups of wilted specimens. However, it is important to note that significant quantitative transmittance variations can be observed even among different fresh specimens belonging to the same
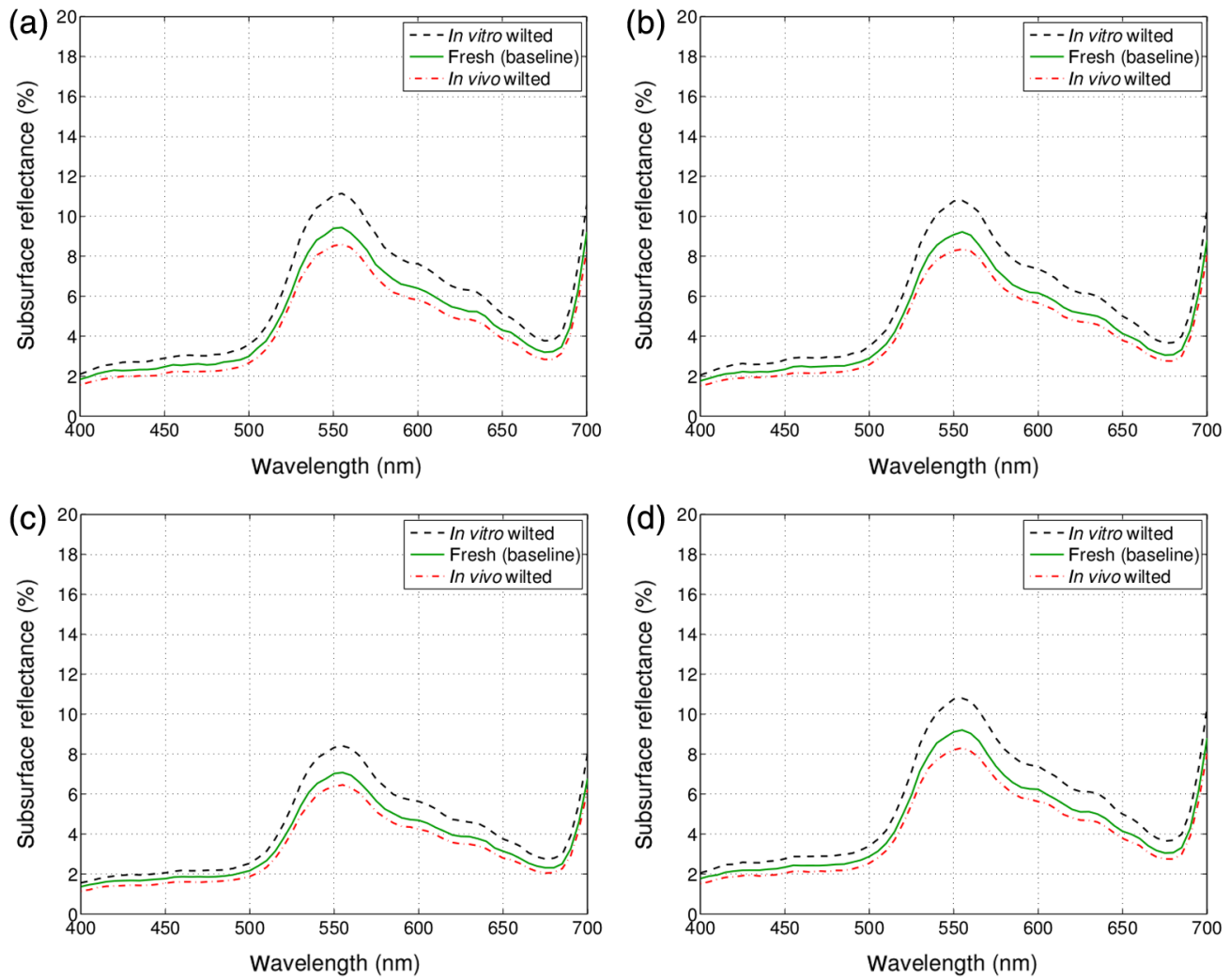

Fig. 2 Subsurface reflectance spectra computed for the maize leaf specimens considering them in fresh and wilted states. The latter correspond to a $25 \%$ reduction in water content. (a) A1, (b) A2, (c) B1, and (d) B2. 

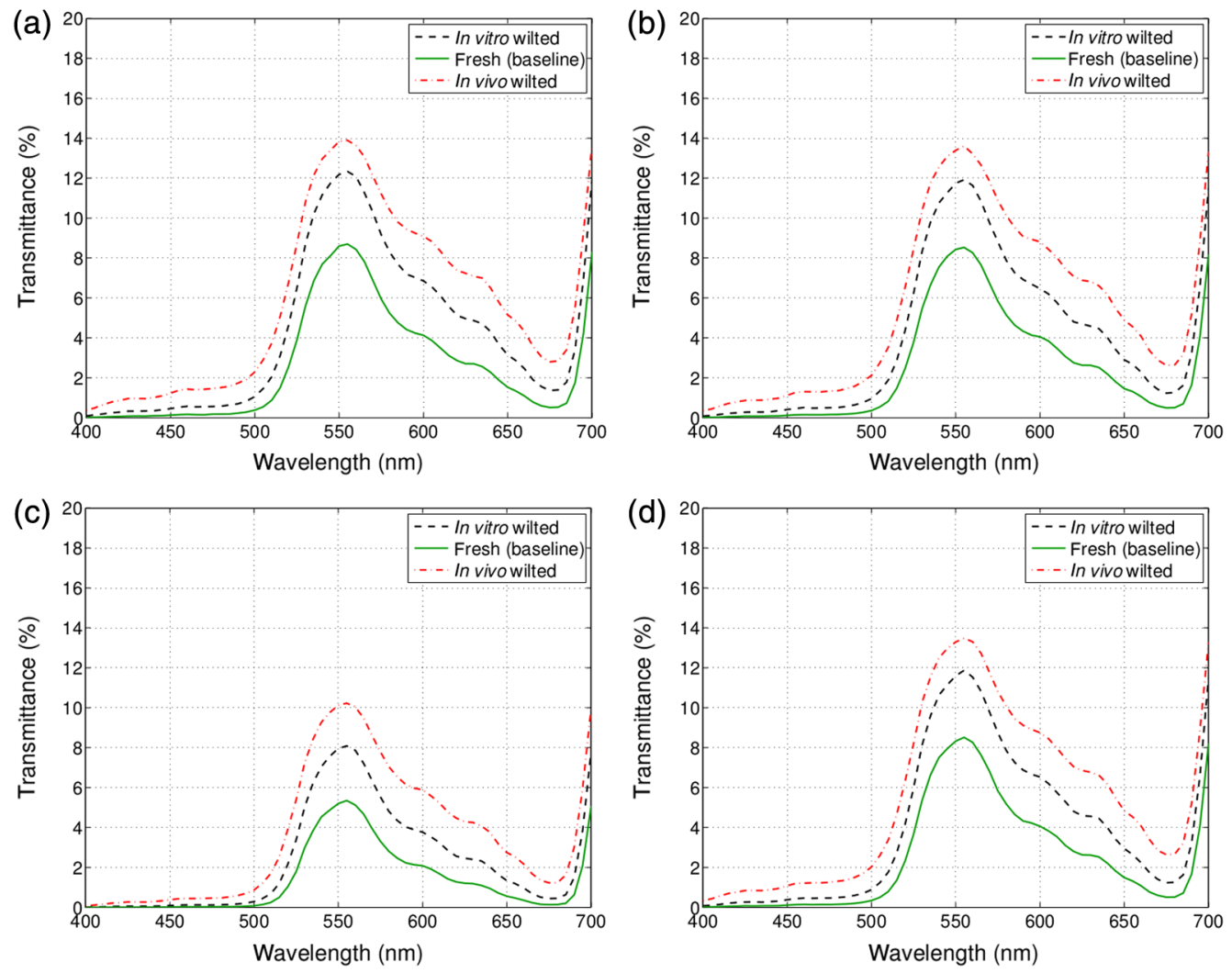

Fig. 3 Transmittance spectra computed for the maize leaf specimens considering them in fresh and wilted states. The latter correspond to a $25 \%$ reduction in water content. (a) A1, (b) A2, (c) B1, and (d) B2.

plant species, as illustrated by the spectral curves presented in Fig. 3. Ideally, one should employ, as an indicator of moderate water stress, a quantity whose interpretation would not be significantly affected by intraspecies biophysical variations. Accordingly, we proceeded to explore the use of subsurface reflectance $\left(\rho_{\mathrm{d}}\right)$ to transmittance $(\tau)$ ratios.

The $\rho_{\mathrm{d}} / \tau$ curves computed for the different groups of specimens are depicted in Fig. 4. As expected, the same qualitative trends can be observed in the curves computed for the four distinct maize leaf specimens. However, one can also observe significant quantitative differences, notably in regions characterized by low transmittance values, namely between 400 to $500 \mathrm{~nm}$ and around $675 \mathrm{~nm}$. These quantitative inconsistencies make $\rho_{\mathrm{d}} / \tau$ values computed for these regions poor indicators of moderate water stress. In addition, it is important to consider that small fluctuations that may occur during the acquisition of transmittance data may lead to larger fluctuations in the computation of the $\rho_{\mathrm{d}} / \tau$ values for these regions.

Possible fluctuations during the acquisition of transmittance data are less likely to affect the computation of the $\rho_{\mathrm{d}} / \tau$ values around $555 \mathrm{~nm}$ since this region is characterized by relatively large subsurface reflectance and transmittance values. In addition, a closer examination of the $\rho_{\mathrm{d}} / \tau$ values within this region reveals a consistent trend for both groups of specimens. More specifically, at $555 \mathrm{~nm}$, the $\rho_{\mathrm{d}} / \tau$ ratio takes values close to 0.6 for the four distinct in vivo wilted specimens, as depicted in Fig. 5. This suggests that the $\rho_{\mathrm{d}} / \tau$ ratio at $555 \mathrm{~nm}$ can be employed as an indicator of moderate water stress in maize, and possibly in other $C_{4}$ species with similar characteristics like sugarcane (Saccharum officinarum), under in vivo conditions. The use of such indicators, in turn, may contribute to the development of new procedures for the reliable estimation of plants' RWC.

Clearly, the use of a $\rho_{\mathrm{d}} / \tau$ value computed at $555 \mathrm{~nm}$ as a moderate water stress indicator, and possibly in RWC estimations, needs to be further investigated through in situ experiments. Moreover, it is also necessary to examine practical aspects involving the acquisition of subsurface reflectance and transmittance data in field campaigns. For example, since actual subsurface 

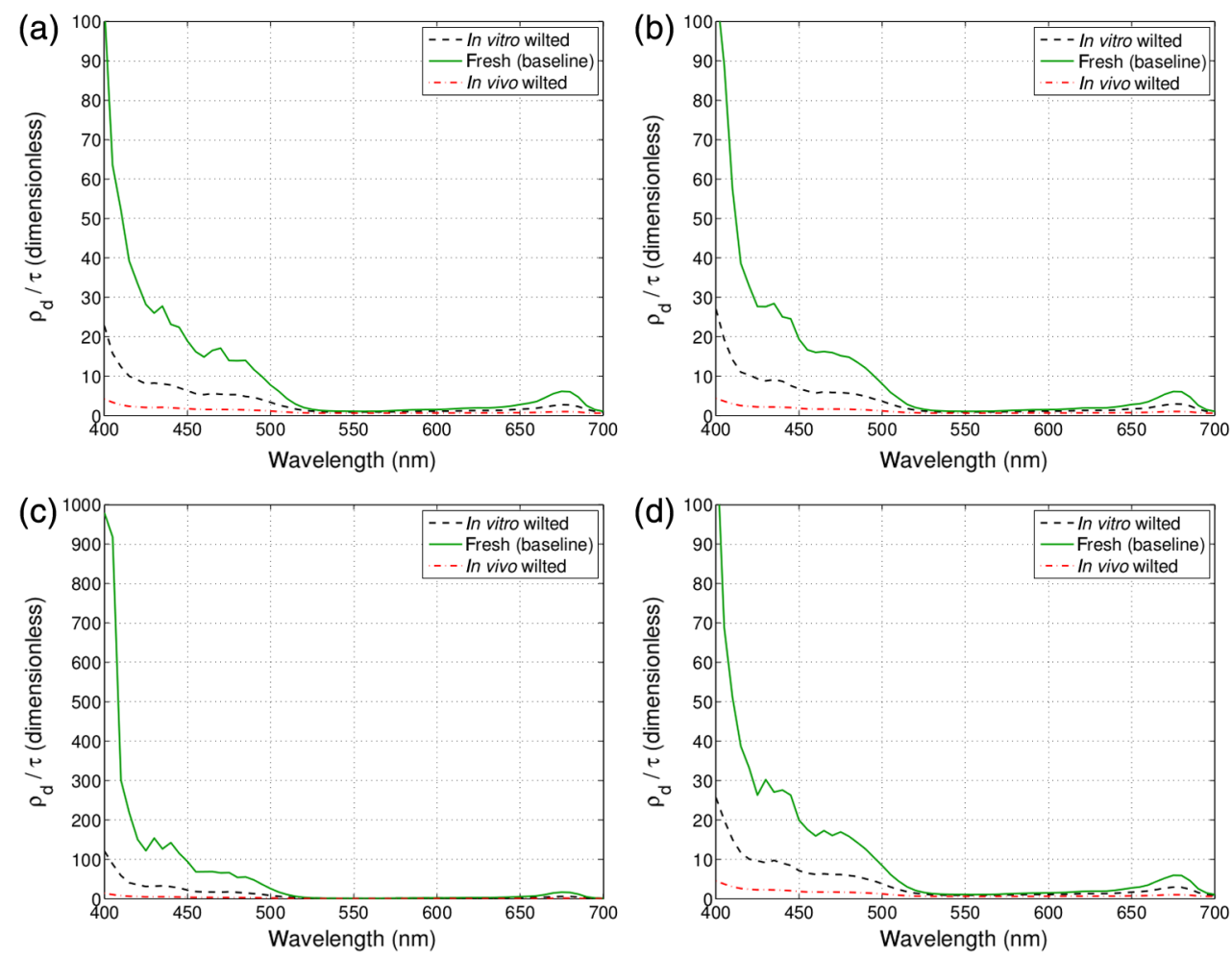

Fig. 4 Ratios of subsurface reflectance $\left(\rho_{\mathrm{d}}\right)$ to transmittance $(\tau)$ computed for the maize leaf specimens considering them in fresh and wilted states. The latter correspond to a $25 \%$ reduction in water content. (a) A1, (b) A2, (c) B1, and (d) B2.
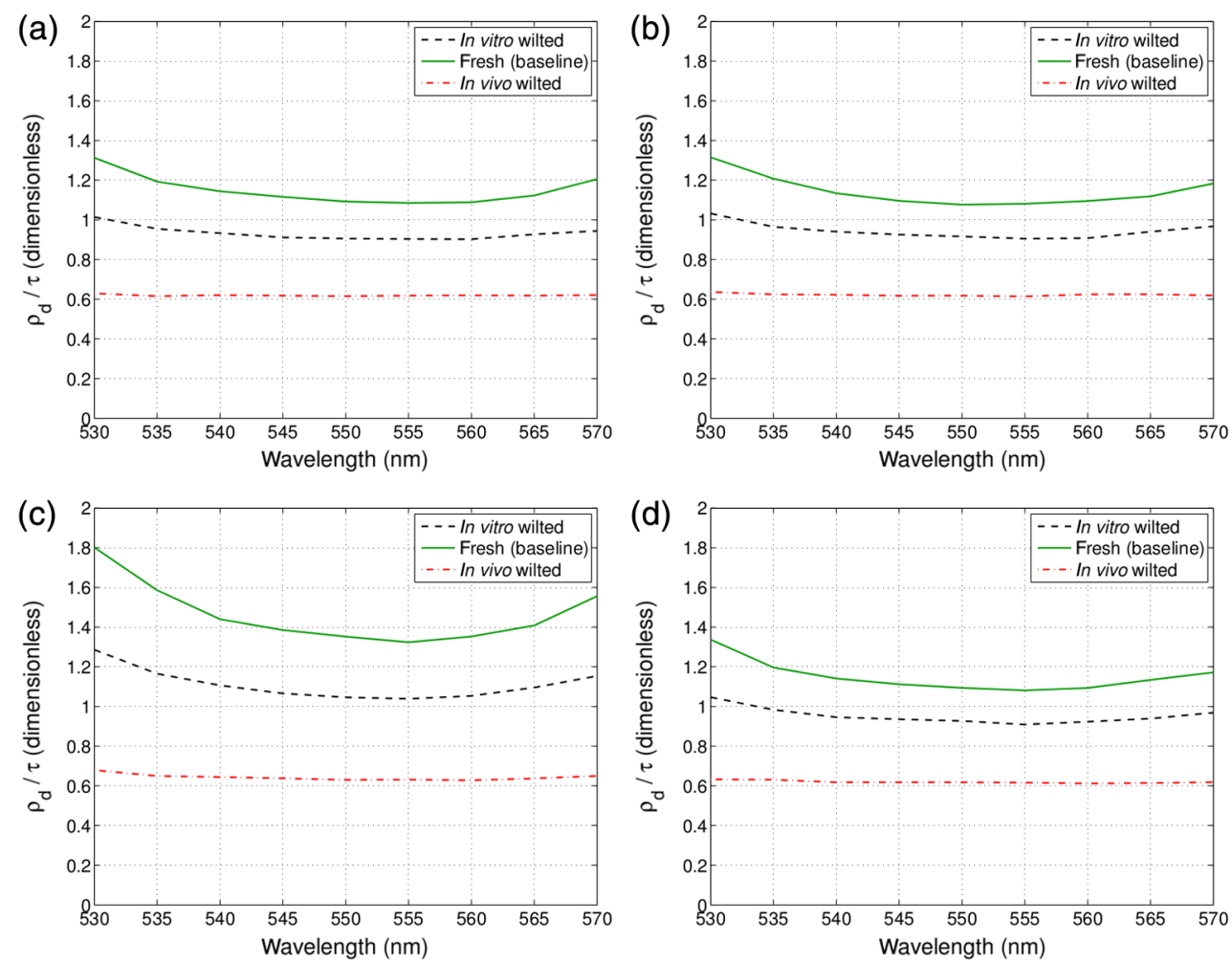

Fig. 5 Zoom-in of the spectral $\rho_{\mathrm{d}} / \tau$ ratios computed for the maize leaf specimens considering them in fresh and wilted states. The latter correspond to a $25 \%$ reduction in water content. (a) A1, (b) A2, (c) B1, and (d) B2. 
reflectance measurements require the use of optical polarization techniques and hardware, ${ }^{23}$ one might wonder whether this requirement might render these measurements impractical outside laboratory settings.

It is worth noting that measured foliar spectral data used in remote sensing investigations are usually obtained at low angles of incidence, with values between 2.5 deg and 1.5 deg being commonly found in the literature..$^{2,3,7,12,32,43}$ In addition, several of these investigations involve separate measurements for the surface and subsurface components of a specimen's reflectance $(\rho)$. It has been reported that surface reflectance contributions are minor for low angles of incidence close to zero. ${ }^{12}$ Furthermore, these contributions result from light interactions at the interface between the air and the cuticle wax layer, ${ }^{44}$ whose optical properties are likely to remain unchanged as a result of moderate water stress. The net effect of these aspects is that, for practical purposes, the relatively small surface reflectance contributions can be treated as a constant under these conditions. Hence, it may be possible to use the reflectance to transmittance ratio as a reliable moderate water stress indicator using $\rho$ and $\tau$ quantities obtained at a low angle of incidence. This would effectively eliminate the need for separating the surface and subsurface components of reflectance through additional optical equipment. Accordingly, we computed $\rho / \tau$ curves in the 530 to $570 \mathrm{~nm}$ range for the different groups of specimens. As can be observed in the plots presented in Fig. 6, at $555 \mathrm{~nm}$, this ratio takes values close to 1.0 for the four distinct in vivo wilted specimens. For completeness, we present in Table 5 the $\rho_{\mathrm{d}} / \tau$ and $\rho / \tau$ values computed for the selected specimens considering the selected angle of incidence $(8 \mathrm{deg})$.

During actual field measurement campaigns, the angle of incidence may vary from one measurement instance to another. For this reason, we have examined the sensitivity of the proposed indicators to such angular variations. We started by reviewing our measurement guidelines. First, the proposed indicators, as envisioned in this work, should be obtained considering a low angle of incidence (e.g., 8 deg as was done in the LOPEX experiments). Hence, assuming that possible angular variations from one measurement to the next will have a small magnitude, we selected $5 \mathrm{deg}$ as the upper bound for the angle of incidence after being affected by these variations. We
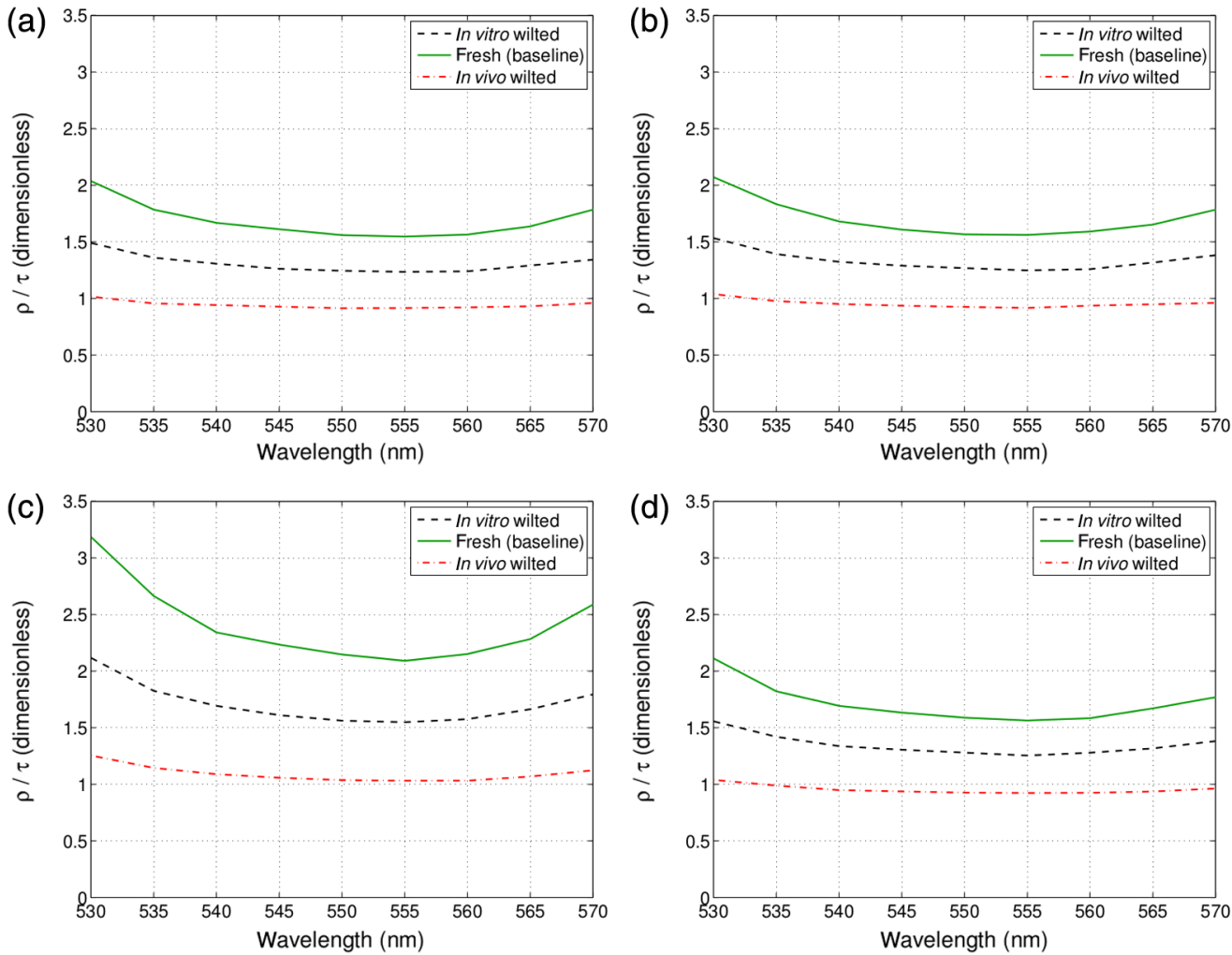

Fig. 6 Ratios of reflectance $(\rho)$ to transmittance $(\tau)$, in the 530 to $570 \mathrm{~nm}$ range, computed for the maize leaf specimens considering them in fresh and wilted states. The latter correspond to a $25 \%$ reduction in water content. (a) $\mathrm{A} 1$, (b) $\mathrm{A} 2$, (c) $\mathrm{B} 1$, and (d) $\mathrm{B} 2$. 
Table 5 Values of $\rho_{\mathrm{d}} / \tau$ and $\rho / \tau$ ratios computed for the maize leaf specimens in their fresh and wilted states. The values were computed $555 \mathrm{~nm}$ and considering an angle of incidence of $8 \mathrm{deg}$. The wilted state corresponds to water stress associated with a $25 \%$ reduction in water content under in vivo conditions.

\begin{tabular}{|c|c|c|c|c|c|c|c|c|}
\hline \multirow[b]{3}{*}{ Ratio } & \multicolumn{8}{|c|}{ Specimens } \\
\hline & \multicolumn{2}{|c|}{$\mathrm{A} 1$} & \multicolumn{2}{|c|}{$\mathrm{A} 2$} & \multicolumn{2}{|c|}{ B1 } & \multicolumn{2}{|c|}{ A2 } \\
\hline & Fresh & Wilted & Fresh & Wilted & Fresh & Wilted & Fresh & Wilted \\
\hline$\rho_{\mathrm{d}} / \tau$ & 1.05 & 0.61 & 1.07 & 0.61 & 1.31 & 0.63 & 1.07 & 0.61 \\
\hline$\rho / \tau$ & 1.51 & 0.91 & 1.55 & 0.91 & 2.08 & 1.02 & 1.55 & 0.92 \\
\hline
\end{tabular}

Table 6 Values of $\rho_{\mathrm{d}} / \tau$ and $\rho / \tau$ ratios computed for the maize leaf specimens in their fresh and wilted states. The values were computed $555 \mathrm{~nm}$ and considering an angle of incidence of 15 deg. The wilted state corresponds to water stress associated with a $25 \%$ reduction in water content under in vivo conditions.

\begin{tabular}{|c|c|c|c|c|c|c|c|c|}
\hline \multirow[b]{3}{*}{ Ratio } & \multicolumn{8}{|c|}{ Specimens } \\
\hline & \multicolumn{2}{|c|}{$\mathrm{A} 1$} & \multicolumn{2}{|c|}{$\mathrm{A} 2$} & \multicolumn{2}{|c|}{ B1 } & \multicolumn{2}{|c|}{$\mathrm{A} 2$} \\
\hline & Fresh & Wilted & Fresh & Wilted & Fresh & Wilted & Fresh & Wilted \\
\hline$\rho_{\mathrm{d}} / \tau$ & 1.09 & 0.63 & 1.11 & 0.63 & 1.37 & 0.64 & 1.11 & 0.63 \\
\hline$\rho / \tau$ & 1.56 & 0.92 & 1.61 & 0.94 & 2.14 & 1.05 & 1.59 & 0.94 \\
\hline
\end{tabular}

then computed the corresponding $\rho_{\mathrm{d}} / \tau$ and $\rho / \tau$ values at $555 \mathrm{~nm}$. As verified in Table 6 , these values remained close to 0.6 and 1.0, respectively. Thus, one can assume that possible small angular variations would not affect the water stress assessment performed using the proposed indicators obtained at low angles of incidence.

In summary, our silico experiments show that unreliable assessments of water status may result from overlooking the adaptation mechanisms developed by certain plants, notably $C_{4}$ species like maize, to cope with adverse environmental conditions. We remark that these adaptation mechanisms, developed to increase a plant's chance of survival, can affect its spectral signature. This is particularly relevant if one aims to prevent irreversible damage to their photosynthetic apparatus, which occurs when the plants' RWC falls below 70\%. According to our in silico experiments, when the specimens' RWC is 5\% away from reaching this physiological limit, the spectral $\rho_{\mathrm{d}} / \tau$ and $\rho / \tau$ ratios computed at $555 \mathrm{~nm}$ and at a low angle of incidence take values close to 0.6 and 1.0, respectively. Hence, we believe that these ratios can serve as references to alert crop managers to take action before a moderate water stress state becomes severe and beyond repair. For example, water stress reduction protocols could be activated when $\left|\rho_{\mathrm{d}}(555) / \tau(555)-0.6\right|<0.1$ or $|\rho(555) / \tau(555)-1.0|<0.1$. In the case of the $\rho / \tau$ ratio values, their computation would be more cost-effective since $\rho$ readings can be obtained from leaf specimens without the use of polarization devices.

It is worth noting that the exposure to moderate water stress conditions may not be uniform across an entire crop, especially in large cultivated areas. Accordingly, the use of supporting ground-based information, such as the indicators examined in this work, to evaluate the plants' water stress status may require a number of in situ measurements. This number, in turn, depends on the geophysical variability of the area, among other factors. Although the costs involved in obtaining supporting ground-based information are directly associated with this number, we believe that they are justifiable considering potential crop yield losses that such data can help to prevent. Further investigations involving field campaigns will be necessary, however, to quantitatively assess this tradeoff. 


\section{Conclusion}

In this work, we have revisited the visible spectral responses of maize specimens to moderate water stress, under both in vitro and in vivo conditions, to explore alternatives for the early detection and monitoring of situations affecting their RWC. The results of our in silico experiments considering four distinct specimens suggest that the subsurface reflectance to transmittance ratio and the total reflectance to transmittance ratio, both measured at $555 \mathrm{~nm}$ and at low angles of incidence (below $15 \mathrm{deg}$ ), may serve as indicators of moderate water stress in $C_{4}$ plants like maize under in vivo conditions. Although further in situ research considering a large number of specimens is necessary to assess the full potential of using leaf spectral responses in the photosynthetic domain to detect and monitor water stress conditions, this possibility is tangible and it may be less constrained by practical issues, such as the use of polarization optics, than was originally expected. As future work, we plan to extend our in silico experiments to a wider range of $C_{4}$ species to assess the generality of the water stress indicators examined in this investigation.

\section{Acknowledgments}

This work was supported by the Natural Sciences and Engineering Research Council of Canada (NSERC-Discovery Grant 237337).

\section{References}

1. R. Gutiérrez, "System biology for enhanced plant nitrogen nutrition," Science 336, 16731675 (2012).

2. J. Thomas et al., "Estimating leaf water content by reflectance measurements," Agron. J. 63, 845-847 (1971).

3. G. Maracci et al., "Interpretation of reflectance spectra by plant physiological parameters," in Int. Geoscience and Remote Sensing Symp. (IGARSS), Espoo, pp. 2303-2306 (1991).

4. J. Verdebout, S. Jacquemoud, and G. Schmuck, "Optical properties of leaves: modeling and experimental studies," in Imaging Spectrometry-A Tool for Environmental Observations, J. Hill and J. Mégier, Eds., pp. 169-191, Kluwer Academic Publishers, Dordrecht (1994).

5. H. Earl and R. Davis, "Effect of drought stress on leaf and whole canopy radiation use efficiency and yield of maize," Agron. J. 95, 688-696 (2003).

6. K. Chenu et al., "Short-term responses of leaf growth rate to water deficit scale up to wholeplant and crop levels: an integrated modelling approach in maize," Plant Cell Environ. 31, 378-391 (2008).

7. G. Baranoski et al., "In silico assessment of environmental factors affecting the spectral signature of $\mathrm{C}_{4}$ plants in the visible domain," Int. J. Remote Sens. 33(4), 1190-1213 (2012).

8. F. Loreto, N. Baker, and D. Ort, "Chloroplast to leaf," Chapter 9 in Photosynthetic Adaptation Chloroplast to Landscape, Part 6: Environmental Constraints, Ecological Studies, W. Smith, T. Vogelmann, and C. Critchley, Eds., Vol. 178, pp. 231-261, Springer, New York (2004).

9. R. Alberte and J. Thornber, "Water stress effects on the content and organization of chlorophyll in mesophyll and bundle sheath chloroplasts of maize," Plant Physiol. 59, 351-353 (1977).

10. G. Hendry, J. Houghton, and S. Brown, "The degradation of chlorophyll-a biological enigma," New Phytol. 107, 255-302 (1987).

11. W. Lee, S. Searcy, and T. Kataoka, "Assessing nitrogen stress in corn varieties of varying color," in ASAE Annual Int. Meeting, Toronto, Ontario, pp. 1-24, ASAE Paper 993034 (1999).

12. J. Woolley, "Reflectance and transmittance of light by leaves," Plant Physiol. 47, 656-662 (1971).

13. G. Carter, "Primary and secondary effects of water content on the spectral reflectance of leaves," Am. J. Botany 78, 916-924 (1991). 
14. A. Yokota, K. Takahara, and K. Akashi, "Water stress," Chapter 2 in Physiology and Molecular Biology of Stress Tolerance in Plants, K. Rao, A. Raghavendra, and K. Reddy, Eds., pp. 15-39, Springer, Dordrecht (2006).

15. M. Schlerf and C. Atzberger, "Imaging spectrometry and vegetation science," in Imaging Spectrometry. Basic Principles and Prospective Applications, F. van der Meer and S. de Jong, Eds., pp. 111-155, Kluwer Academic Publishers, Dordrecht (2001).

16. Y. Sato, M. Wada, and A. Kadota, "External $\mathrm{Ca}^{2+}$ is essential for chloroplast movement induced by mechanical stimulation but not by light stimulation," Plant Physiol. 127, 497-504 (2001).

17. I. Terashima and T. Saeki, "Light environment within a leaf I. Optical properties of paradermal sections of camelia leaves with spectral reference to differences in the optical properties of palisade and spongy tissues," Plant Cell Physiol. 24, 1493-1501 (1983).

18. T. Vogelmann, "Plant tissue optics," Annu. Rev. Plant Physiol. Plant Mol. Biol. 44, 231-251 (1993).

19. M. Govender et al., "Review of commonly used remote sensing and ground-based technologies to measure plant water stress," Water SA 35(5), 741-752 (2009).

20. L. Liu et al., "Detection of leaf and canopy EWT by calculating REWT from reflectance spectra," Int. J. Remote Sens. 31(10), 2681-2695 (2010).

21. S. Dobrowski et al., "Simple reflectance indices track heat and water stress-induced changes in steady-state chlorophyll fluorescence at the canopy scale," Remote Sens. Environ. 97, 403-414 (2005).

22. G. Baranoski et al., "On the high-fidelity monitoring of $\mathrm{C} 3$ and $\mathrm{C} 4$ crops under nutrient and water stress," Proc. SPIE 8524, 85240W (2012).

23. V. Vanderbilt, C. Daughtry, and R. Dahlgren, "Relative water content, bidirectional reflectance and bidirectional transmittance of the interior of detached leaves during dry down," in IEEE Int. Geoscience and Remote Sensing Symp. (IGARSS), Abstract (2015).

24. B. Li-Ping et al., "Effect of soil drought stress on leaf water stress, membrane permeability and enzymatic antioxidant system of maize," Pedosphere 16(3), 326-332 (2006).

25. G. Baranoski, S. Van Leeuwen, and T. Chen, "On the decomposition of foliar hyperspectral signatures for the high-fidelity discrimination and monitoring of crops," Proc. SPIE 9880, 98800G (2016).

26. G. Baranoski, "Modeling the interaction of infrared radiation (750 to $2500 \mathrm{~nm}$ ) with bifacial and unifacial plant leaves," Remote Sens. Environ. 100, 335-347 (2006).

27. G. Baranoski and D. Eng, "An investigation on sieve and detour effects affecting the interaction of collimated and diffuse infrared radiation (750 to $2500 \mathrm{~nm}$ ) with plant leaves," IEEE Trans. Geosci. Remote Sens. 45, 2593-2599 (2007).

28. D. Eng and G. Baranoski, "The application of photoacoustic absorption spectral data to the modeling of leaf optical properties in the visible range," IEEE Trans. Geosci. Remote Sens. 45, 4077-4086 (2007).

29. A. Krishnaswamy and G. Baranoski, "A biophysically-based spectral model of light interaction with human skin," Comput. Graphics Forum 23(3), 331-340 (2004).

30. G. Baranoski and J. Rokne, Light Interaction with Plants: A Computer Graphics Perspective, Horwood Publishing, Chichester (2004).

31. G. Baranoski, J. Rokne, and G. Xu, "Virtual spectrophotometric measurements for biologically and physically-based rendering," Visual Comput. 17(8), 506-518 (2001).

32. B. Hosgood et al., "Leaf optical properties experiment 93," Tech. Rep. Report EUR 16095 EN, Joint Research Center, European Comission, Institute for Remote Sensing Applications, Luxembourg (1995).

33. NPSG, "Run ABM-U online," Natural Phenomena Simulation Group, University of Waterloo, Canada (2011), http://www.npsg.uwaterloo.ca/models/ABMU.php

34. G. Baranoski et al., "Rapid dissemination of light transport models on the web," IEEE Comput. Graphics Appl. 32(3), 10-15 (2012).

35. NPSG, "Plant leaf data," Natural Phenomena Simulation Group, University of Waterloo, Canada (2011), http://www.npsg.uwaterloo.ca/data/leaves.php

36. T. Vogelmann and G. Martin, "The functional significance of palisade tissue: penetration of directional versus diffuse light," Plant Cell Environ. 16, 65-72 (1993). 
37. B. Bowes, A Colour Atlas of Plant Structure, pp. 97-116, Manson Publishing Ltd., London (1996).

38. J. Woolley, "Change of leaf dimensions and air volume with change in water content," Plant Physiol. 41, 815-816 (1973).

39. E. Rabinowitch, "Light absorption by pigments in the living cell," in Photosynthesis and Related Processes, Vol. 2, pp. 672-739, Interscience Publishers, Inc., New York, Part 1 (1951).

40. J. Evans, T. Vogelmann, and W. Williams, "Chloroplast to leaf," Chapter 2 in Photosynthetic Adaptation Chloroplast to Landscape, Part 2: Sunlight Capture, Ecological Studies, W. Smith, T. Vogelmann, and C. Critchley, Eds., Vol. 178, pp. 15-41, Springer, New York (2004).

41. L. Björn, "Interception of light by plants leaves," Chapter 11 in Crop Photosynthesis: Spatial and Temporal Determinants, N. Baker and H. Thomas, Eds., pp. 253-276, Elsevier Science Publishers, New York (1992).

42. L. Fukshansky, "Optical properties of plants," in Plants and the Daylight Spectrum, H. Smith, Ed., pp. 21-40, Academic Press, London (1981).

43. A. Al-Abbas et al., "Spectra of normal and nutrient-deficient maize leaves," Agron. J. 66, 16-20 (1974).

44. V. Vanderbilt, L. Grant, and S. Ustin, "Polarization of light by vegetation," in PhotonVegetation Interactions: Applications in Optical Remote Sensing and Ecology, R. Nynemi and J. Ross, Eds., pp. 191-228, Springer Verlag, Berlin (1991).

Gladimir V. G. Baranoski obtained his PhD in computer science at the University of Calgary (Canada) in 1998. Currently, he is a faculty member of the David R. Cheriton School of Computer Science at the University of Waterloo (Canada), where he has established the Natural Phenomena Simulation Group. His research interests primarily include the predictive simulation of light interactions with natural materials aimed at interdisciplinary applications. $\mathrm{He}$ is a member of SPIE.

Spencer R. Van Leeuwen is currently pursuing his master's degree in computer science at the David R. Cheriton School of Computer Science at the University of Waterloo (Canada). He holds a bachelor of mathematics degree in computer science and combinatorics and optimization with a minor in music from the same institution. He is also a member of the Natural Phenomena Simulation Group at the University of Waterloo. 